Mots. Les langages du politique

\title{
ALDRIN Philippe, HUBÉ Nicolas, OLLIVIER-YANIV Caroline et UTARD Jean-Michel, Les mondes de la communication publique. Légitimation et fabrique symbolique du politique
}

Rennes, Presses universitaires de Rennes, 2014, 190 pages, $18 €$

Pierre-Emmanuel Guigo

\section{OpenEdition}

\section{Journals}

\section{Édition électronique}

URL : https://journals.openedition.org/mots/22405

DOI : $10.4000 /$ mots. 22405

ISBN : 978-2-84788-836-2

ISSN : 1960-6001

Éditeur

ENS Éditions

\section{Édition imprimée}

Date de publication : 10 septembre 2016

Pagination : 147-150

ISBN : $978-2-84788-835-5$

ISSN : 0243-6450

Référence électronique

Pierre-Emmanuel Guigo, « ALDRIN Philippe, HUBÉ Nicolas, OLLIVIER-YANIV Caroline et UTARD JeanMichel, Les mondes de la communication publique. Légitimation et fabrique symbolique du politique», Mots. Les langages du politique [En ligne], 111 | 2016, mis en ligne le 10 septembre 2016, consulté le 22 avril 2022. URL : http://journals.openedition.org/mots/22405; DOI : https://doi.org/10.4000/mots. 22405 


\title{
Compte rendu de lecture
}

\author{
Les mondes de la communication publique. \\ Légitimation et fabrique symbolique du politique \\ Philippe Aldrin, Nicolas Hubé, Caroline Ollivier-Yaniv, Jean-Michel Utard. \\ Rennes, Presses universitaires de Rennes, 2014, 190 pages, $18 €$
}

Si la communication politique suscite depuis longtemps l'intérêt des chercheurs, la communication publique est un champ nouveau de la recherche en plein développement. C'est ce que permet de voir cet ouvrage collectif, issu de plusieurs rencontres et colloques en France et au Québec. Inspiré par une vision «critique» de la communication, articulée autour de la notion de «domination » - voir Pierre Bourdieu ${ }^{1}$-, l'ensemble de l'ouvrage ne prend pas la notion de communication publique comme allant de soi. Il décortique au contraire le discours dominant, en particulier celui des promoteurs de cette discipline, soulignant l'inefficacité d'une dichotomie communication publique/communication politique. La communication publique n'est pas la communication institutionnelle et neutre que voulaient théoriser ses promoteurs. Elle n'est pas détachée d'intérêts purement politiques et de rapports de force. Bien loin des discours légitimants, toutes les contributions montrent que la frontière entre communication publique et communication politique est plus qu'ambiguë. Jérémie Nollet propose d'ailleurs une graduation entre les deux termes plutôt qu'une opposition.

Une première partie est dédiée à une analyse diachronique de la communication publique et de ses évolutions. Cette généalogie permet de montrer que cette institutionnalisation de la communication publique n'est pas complète, les frontières sont toujours mouvantes. La définition identitaire des acteurs n'est pas encore acquise, comme le souligne Jean-Baptiste Legavre dans sa contribution ("Se trouver un nom de métier : le cas des "communicants publics" ») soulignant l'évolution du qualificatif, et son adoption ou rejet suivant les représentations que portent les acteurs.

Mais ce premier chapitre permet néanmoins de montrer l'emprise croissante de la communication sur l'activité politique et en particulier l'État. Jérémie Nollet ("L'économie hybride de la "communication de crise" : une cristallisation des principes contradictoires de la communication ministérielle») montre ainsi comment des crises sanitaires comme celle de la vache folle obligent à une réaction en termes de communication et à une adaptation aux

1. Que l'on peut opposer, avec Arnaud Mercier (Le journal télévisé : politique de l'information et information politique, Paris, Presses de Sciences-Po, 1996, p. 120), à la thèse de la «démocratisation » portée notamment par Jean-Louis Missika ou Pippa Norris. 
pratiques journalistiques. Une stratégie de communication de crise, inspirée du monde de l'entreprise, se met dès lors en œuvre pour répondre à ces situations.

Les collectivités territoriales sont aussi touchées et, selon Christian Le Bart ("La communication des collectivités locales : les territoires entre exemplarité, standardisation, et singularité»), elles en sont même une proie encore plus facile. Dans un espace de plus en plus concurrentiel, il devient nécessaire pour elles de mettre en place des stratégies de valorisation des politiques publiques locales, et non pas seulement des identités prescrites comme d'ordinaire (situation géographique, climat, histoire, etc.). Ce phénomène s'accompagne du paradoxe suivant, souligné par l'auteur : ces politiques de différenciation sont en général toujours les mêmes et amènent ainsi leur standardisation et même leur désidéologisation à l'échelle du territoire, en vue de s'adapter à un monde conçu sur le mode du réseau et de la communication.

Une deuxième partie est plus centrée sur des études de cas contemporains qui soulignent la «coproduction» de la communication publique entre État, collectivités territoriales, société civile.

Pascal Dauvin («Quand l'environnement et la participation deviennent des instruments de la communication événementielle : l'exemple de la première journée sans voiture à La Rochelle) montre comment cet événement fut coproduit par les communicants (ici, le service de communication de la mairie de La Rochelle), les politiques (le maire Michel Crépeau) et les médias, s’appuyant notamment sur une procédure de participation qui servit à légitimer l'événement et à taire les critiques.

Guillaume Gourgues («Communiquer, une opération politique plurielle et stratégique: le cas de la démocratie participative en Bourgogne ») nuance l'opposition entre communication et participation. La communication est nécessaire à la mise en œuvre d'une procédure participative, surtout lorsque celleci est mise en place par les politiques et selon des thématiques édictées par ceux-ci. Par ailleurs, la participation, comme dans le cas rochelais de la contribution précédente, est aussi un moyen de communication favorisant l'image d'une région (ici, la Bourgogne) comme terre de démocratie participative. II souligne ainsi les usages politiques de ces débats participatifs dans les rapports de force locaux (voire entre local et national), et tout particulièrement entre les différents courants du PS (entre le courant majoritaire du PS et le NPS autour de Christian Paul).

Mais la société civile n'est pas complètement absente de la communication politique, comme le montre Jean-Philippe de Oliveira à partir de l'exemple des campagnes de prévention contre le sida ("Quand les associations coproduisent l'action publique : enjeux et tension autour des campagnes grand public de prévention du sida »). Il souligne ainsi la capacité des associations - et en particulier ici Aides et Act Up - à influencer les modes de communi- 
cation (notamment vers une explicitation des pratiques homosexuelles) des campagnes publiques, sans pour autant participer à celles-ci directement. Ces campagnes qui semblent pourtant directement dirigées par l'État sont en fait un lieu de négociation entre État et société civile, les associations étant indispensables pour l'État en raison de l'expertise qu'elles ont acquise dans ce domaine et par leur capacité à faire remonter les problématiques de la base.

Mais un modèle plus général de communication s'impose au sein des différents territoires, notamment du point de vue lexical. C'est ce que montrent Sarah Labelle et Claire Oger à partir du cas du Grand Lyon ("Les institutions culturelles publiques à l'épreuve de la gouvernance : communication et reconfiguration du politique »). Le discours de la gouvernance tend à s'imposer dans la communication de l'agglomération, même dans le domaine de la culture, afin de se fondre dans un moule plus global et se montrer compétitif. La culture est ainsi présentée comme étant au service de l'économie et doit avoir une valeur créative.

Pour finir, l'ouvrage postule une thèse "continuiste». Pour les auteurs, il faut à tout prix éviter l'écueil consistant à voir dans chaque innovation ou chaque stratégie de communication une pure nouveauté, et donc à croire que tout s'expliquerait par la communication. Leurs contributions soulignent ainsi que si les formes ont certes changé, le fond politique est toujours celui de la démocratie libérale telle qu'elle s'est formalisée depuis le xıxe siècle. Mais il faudrait aussi éviter le Scylla de l'immobilité qui tendrait à refuser toute évolution. Pour les auteurs, il faut ainsi éclairer les évolutions en les contextualisant et en les mettant en perspective. Par une analyse associant diachronie et diatopie, jeu des acteurs et évolutions des idées (imprégnation croissante par l'idéologie libérale et l'idée de mondialisation), l'ouvrage propose ainsi des bases et une méthodologie pour les recherches à venir.

Pierre-Emmanuel Guigo

IEP de Paris, Centre d'histoire 